\title{
Tumor Response Criteria in Oncoimaging: RECIST Criteria and beyond-Part 2
}

\author{
${ }^{1}$ Department of Radiodiagnosis, Tata Memorial Hospital, Mumbai, \\ Maharashtra, India \\ 2Department of Radiodiagnosis, Delhi State Cancer Institute, \\ New Delhi, India \\ ${ }^{3}$ Department of Nuclear Medicine, Tata Memorial Hospital, Mumbai, \\ Maharashtra, India \\ ${ }^{4}$ Department of Imaging, Dana Farber Cancer Institute, Harvard \\ Medical School, Boston, Massachusetts, United States \\ ${ }^{5}$ Department of Radiology, Brigham and Women's Hospital, \\ Harvard Medical School, Boston, Massachusetts, United States
}

Akshay D. Baheti ${ }^{1}$ Ashita Rastogii ${ }^{2, \odot}$ Aravintho Natarajan $^{3}$ Anurima Patra ${ }^{1}$ Sree Harsha Tirumani ${ }^{4,5}$

Address for correspondence Ashita Rastogi, DNB (Radiodiagnosis), Fellowship in Cancer Imaging, Department of Radiodiagnosis, Delhi State Cancer Institute, Dilshad Garden, New Delhi 110095, India (e-mail: ashitarastogi@hotmail.com).

J Gastrointestinal Abdominal Radiol ISGAR 2019;2:107-115

\begin{abstract}
Keywords

- treatment response

- modified RECIST

- Choi criteria

- MD Anderson criteria

- PERCIST

- immune RECIST

The rapid recent advances in oncology have made the dream of precision oncology a reality, with targeted therapy available for various tumors depending on the molecular genotype. This has led to the corresponding development of personalized radiology as well, with various tumor response criteria used to characterize disease response/ progression depending on chemotherapy used. In these two review articles, we review the various tumor response criteria widely applied in both research and clinical settings. These include the classic size-based criteria such as RECIST (Response Evaluation Criteria In Solid Tumors) 1.1 and the WHO (World Health Organization) criteria, as also various other criteria such as Choi and modified Choi criteria for tumors treated by targeted therapy, EASL (European Association for the Study of the Liver) and modified RECIST (mRECIST) criteria for hepatocellular carcinomas, immune-related response criteria (irRC) and immune RECIST (iRECIST) for patients on immunotherapy. Other clinically important criteria such as PERCIST (PET Response Criteria In Solid Tumors) for positron emission tomography-computed tomography (PET-CT), and the MD Anderson criteria for evaluating bone metastases are also highlighted.
\end{abstract}

\section{Introduction}

In our previous article, we discussed the classic size-based RECIST criteria that are used in most clinical trials to assess tumor response to therapy. In this second article, we discuss various alternative response assessment criteria that can be used instead of RECIST (Response Evaluation Criteria In Solid Tumors) in specific situations depending on the primary tumor, site of disease, and the chemotherapy regimen used.

Over the past decade or so, numerous novel anticancer medications have been approved, many of which have different mechanisms of action as compared with cytotoxic or cytostatic conventional chemotherapy. These include

received

March 3, 2019

accepted after revision

March 26, 2019

published online

August 6, 2019 targeted chemotherapy such as imatinib and VEGF (vascular endothelial growth factor) inhibitors (such as sunitinib) and immunotherapy such as ipilimumab and nivolumab. These drugs have heralded the onset of personalized medicine and its offshoot, personalized radiology. These novel treatments often incite a tumor response that is different from the conventional reduction in size. As a result, RECIST criteria may not correctly classify tumor response in these cases, and different alternative response criteria have developed to more accurately assess patients on these drugs. Similarly, RECIST criteria are not sufficiently accurate to assess tumors such as hepatocellular carcinoma (HCC) and lymphomas, or sites such as bone metastases, nor do they incorporate
License terms

Gastrointestinal and Abdominal Radiology

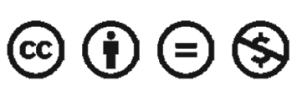


Table 1 Alternative response criteria

\begin{tabular}{|c|c|c|c|c|}
\hline $\begin{array}{l}\text { Response } \\
\text { categories }\end{array}$ & Choi criteria & mRECIST & MDA criteria for bone & PERCIST \\
\hline CR & $\begin{array}{l}\text { Disappearance of all } \\
\text { lesions and no new } \\
\text { lesions }\end{array}$ & $\begin{array}{l}\text { Disappearance of } \\
\text { any intratumoral } \\
\text { arterial } \\
\text { enhancement in all } \\
\text { target lesions }\end{array}$ & $\begin{array}{l}\text { Complete sclerotic fill-in of lytic } \\
\text { lesions on radiographs or CT } \\
\text { Complete disappearance of hot } \\
\text { spots on scintigraphy or of tumor } \\
\text { signal on MRI } \\
\text { Normalization of bone density on } \\
\text { radiographs or CT }\end{array}$ & $\begin{array}{l}\text { Visual disappearance of } \\
\text { all metabolically active } \\
\text { tumors, i.e., normaliza- } \\
\text { tion of the SUL to less } \\
\text { than mean liver SUL } \\
\text { and equal to normal } \\
\text { surrounding SUL }\end{array}$ \\
\hline PR & $\begin{array}{l}\text { Decrease in tumor size } \\
\geq 10 \% \text { or a decrease in } \\
\text { tumor attenuation } \geq \\
15 \% \text { at } C T, \text { with no new } \\
\text { lesions and no obvious } \\
\text { progression of non- } \\
\text { measurable disease }\end{array}$ & $\begin{array}{l}\text { At least a } 30 \% \text { de- } \\
\text { crease in the sum of } \\
\text { diameters of viable } \\
\text { (enhancement in } \\
\text { the arterial phase) } \\
\text { target lesions }\end{array}$ & $\begin{array}{l}\text { Development of a sclerotic rim } \\
\text { around lytic lesions on radiographs } \\
\text { or CT or sclerosis of a previously } \\
\text { undetected lesion } \\
\text { Partial sclerosis or fill-in of lytic } \\
\text { lesions on radiographs or CT } \\
\geq 50 \% \text { decrease in measurable } \\
\text { lesions on radiographs, CT, or MRI } \\
\geq 50 \% \text { subjective decrease in tracer } \\
\text { uptake on scintigraphy } \\
\geq 50 \% \text { decrease in sizes of blastic } \\
\text { lesions on radiographs or CT }\end{array}$ & $\begin{array}{l}\text { > a } 30 \% \text { and a } 0.8 \text {-unit } \\
\text { decline in SULpeak } \\
\text { between the most } \\
\text { intense lesion before } \\
\text { treatment and the most } \\
\text { intense lesion after } \\
\text { treatment, although not } \\
\text { necessarily the same } \\
\text { lesion }\end{array}$ \\
\hline PD & $\begin{array}{l}\text { Increase in tumor size } \\
\geq 10 \% \text {, which does not } \\
\text { meet the criteria for } \\
\text { partial response by } \\
\text { tumor attenuation CT } \\
\text { and/or new lesions. } \\
\text { (New lesions include } \\
\text { new areas of enhance- } \\
\text { ment within the tumor } \\
\text { with no change in } \\
\text { overall dimensions) }\end{array}$ & $\begin{array}{l}\text { An increase of at } \\
\text { least } 20 \% \text { in the sum } \\
\text { of the diameters of } \\
\text { viable (enhancing) } \\
\text { target lesions, taking } \\
\text { as reference the } \\
\text { smallest sum of the } \\
\text { diameters of viable } \\
\text { (enhancing) target } \\
\text { lesions recorded since } \\
\text { treatment started }\end{array}$ & $\begin{array}{l}\geq 25 \% \text { increase in size of any mea- } \\
\text { surable lesions on radiographs, CT, } \\
\text { or MRI } \\
\geq 25 \% \text { increase in activity on } \\
\text { scintigraphy } \\
\text { New bone metastases on } \\
\text { scintigraphy, radiographs, CT, or } \\
\text { MRI }\end{array}$ & $\begin{array}{l}\text { > a } 30 \% \text { and } 0.8 \text {-unit } \\
\text { increase in SULpeak } \\
\text { or new lesions, if } \\
\text { confirmed. }(A>75 \% \\
\text { increase in total lesion } \\
\text { glycolysis is proposed } \\
\text { as another metric of } \\
\text { progression) }\end{array}$ \\
\hline SD & Neither of the above & $\begin{array}{l}\text { Change in lesion(s) } \\
\text { does not qualify for } \\
\text { either PR or PD }\end{array}$ & $\begin{array}{l}\text { No change } \\
<25 \% \text { increase or }<50 \% \text { decrease in } \\
\text { size of measurable lesions } \\
\text { No new bone metastases }\end{array}$ & $\begin{array}{l}\text { Does not meet other } \\
\text { criteria }\end{array}$ \\
\hline
\end{tabular}

Abbreviations: CR, complete response; CT, computed tomography; MDA, MD Anderson; mRECIST, modified Response Evaluation Criteria In Solid Tumors; MRI, magnetic resonance imaging; PD, progressive disease; PET, positron emission tomography; PERCIST, PET Response Criteria In Solid Tumors; PR, partial response; SD, stable disease; SULpeak, peak standardized uptake value normalized to lean body mass; WHO, World Health Organization.

modalities such as positron emission tomography-computed tomography (PET-CT) completely. We discuss the various alternative response criteria (-Table $\mathbf{1}$ ) that can be used in such specific situations, so as to successfully provide personalized radiology reports based on the patient's treatment.

\section{Therapy-Specific Criteria \\ Choi Criteria}

Originally described for imatinib response assessment in patients with gastrointestinal stromal tumors (GISTs), these criteria were landmark as they heralded the advent of personalized radiology and alternative response criteria and will hence be discussed in detail. GIST is an extremely chemoresistant tumor that demonstrated a remarkable response to imatinib therapy in early trials. However, the response was unique as compared with conventional chemotherapy as there was often little initial shrinkage in the tumor size, but with dramatic decrease in tumor enhancement and inhomogeneity. ${ }^{1,2}$ The tumors appeared well defined, homogeneous, and hypodense (almost cystic in appearance) after initiation of treatment with imatinib. This is due to the development of cystic or myxoid change within the tumor without significant necrosis or inflammation. Tumor response was also confirmed by significant decrease in the FDG uptake on the tumors on PET-CT.

Choi hence proposed the new criteria that incorporated tumor attenuation along with tumor size to accurately assess response (-Fig. 1). ${ }^{3}$ Given that GIST did not shrink significantly, the "RECIST cutoff" of $30 \%$ decrease in size was lowered to $10 \%$, and a $\geq 15 \%$ decrease in attenuation was also proposed to signify response. Development of resistance to imatinib also follows a similar pathway, with the development of new foci of enhancement within a previously hypoattenuating lesion (nodule-within-cyst appearance) often being the first sign of progression. ${ }^{2,4}$ A $10 \%$ increase in the sum of diameters was also proposed to signify disease progression. These criteria were found to be more sensitive and correlated better with disease-specific survival. ${ }^{3}$

Choi criteria were path breaking as dedicated criteria because a specific tumor treated with a specific therapy was proposed for the first time, paving the way for personalized radiology. The concept that factors beyond tumor 


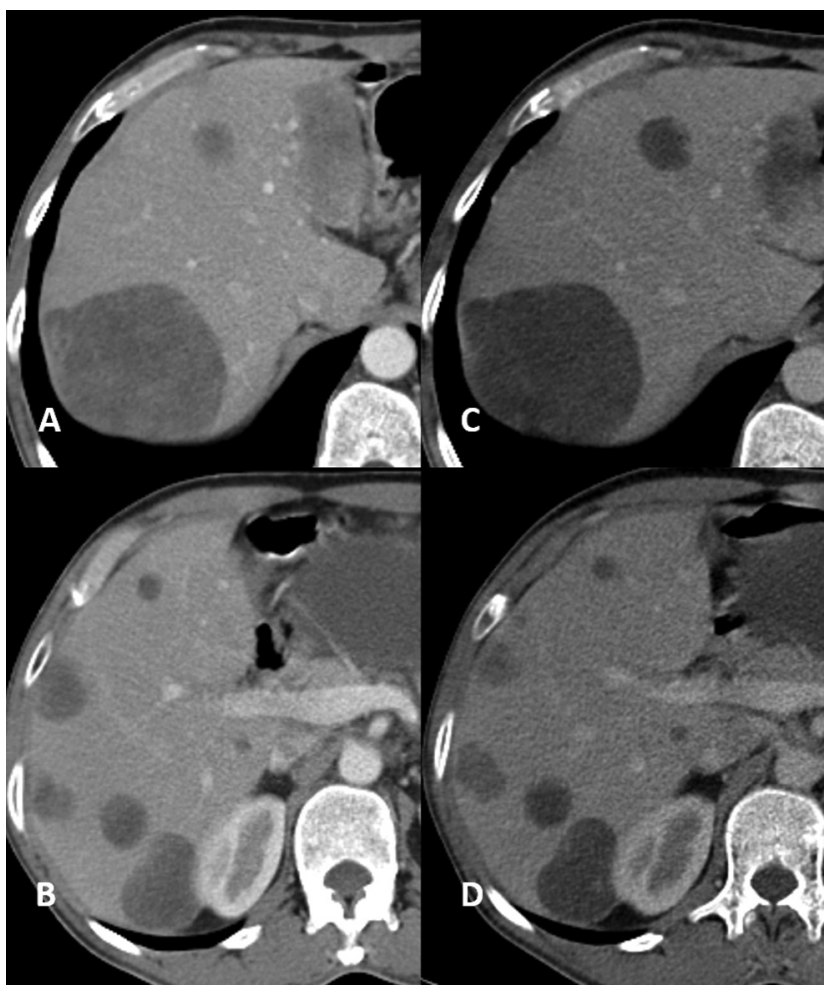

Fig. 1 The patient with gastric GIST and liver metastases, pre (A, B) and post $(\mathbf{C}, \mathbf{D})$ imatinib therapy. The baseline scan shows heterogeneous liver metastases, which become well-demarcated and hypoattenuating on the posttreatment $\mathrm{CT}$, with no significant change in size. Note that the seg IVA lesion appears mildly increased in size because the initially isoattenuating periphery of the metastasis has now become hypoattenuating (pseudoprogression). This would be considered stable disease by RECIST criteria but should be classified as partial response using Choi criteria. size can be objectively assessed (tumor density in this case) was also introduced for the first time. While classically applied to patients with GIST on imatinib, these criteria can also be used in assessing response in patients on other targeted therapies such as VEGF inhibitors, which may also demonstrate similar responses.

\section{Immune-Related Response Criteria and Immune RECIST}

The advent of immunotherapy has been the next big step in cancer treatment. Unlike conventional chemotherapy that has direct cytotoxic or cytostatic effects, immunotherapy works by inciting an immune response by the patient's own immune system against the "foreign" cancer cells. These immunomodulatory drugs activate the host immunity, leading to an inflammatory response against the tumor cells. The peritumoral inflammation and edema that consequently happen may lead to an apparent increase in the tumor size if imaged at this time point (pseudoprogression), as computed tomography (CT) will not be able to differentiate between the lesion and associated edema, measuring them together. Subsequently, the host's immunity will kill the cancer cells leading to tumor shrinkage. ${ }^{5,6}$ Similarly, a previously microscopic lesion (and hence not visualized on CT) may become "newly" apparent due to the associated host response and may be falsely considered a new lesion (-Fig. 2). ${ }^{7}$ Both these scenarios would be labeled as progressive disease as per RECIST criteria but could actually represent early phase of response. Hence, to overcome the shortcomings of RECIST, Wolchok et $\mathrm{al}^{8}$ proposed the immune-related response criteria (irRC) in 2009 for patients on immunotherapy using bidimensional measurements based on the WHO (World Health Organization) criteria.

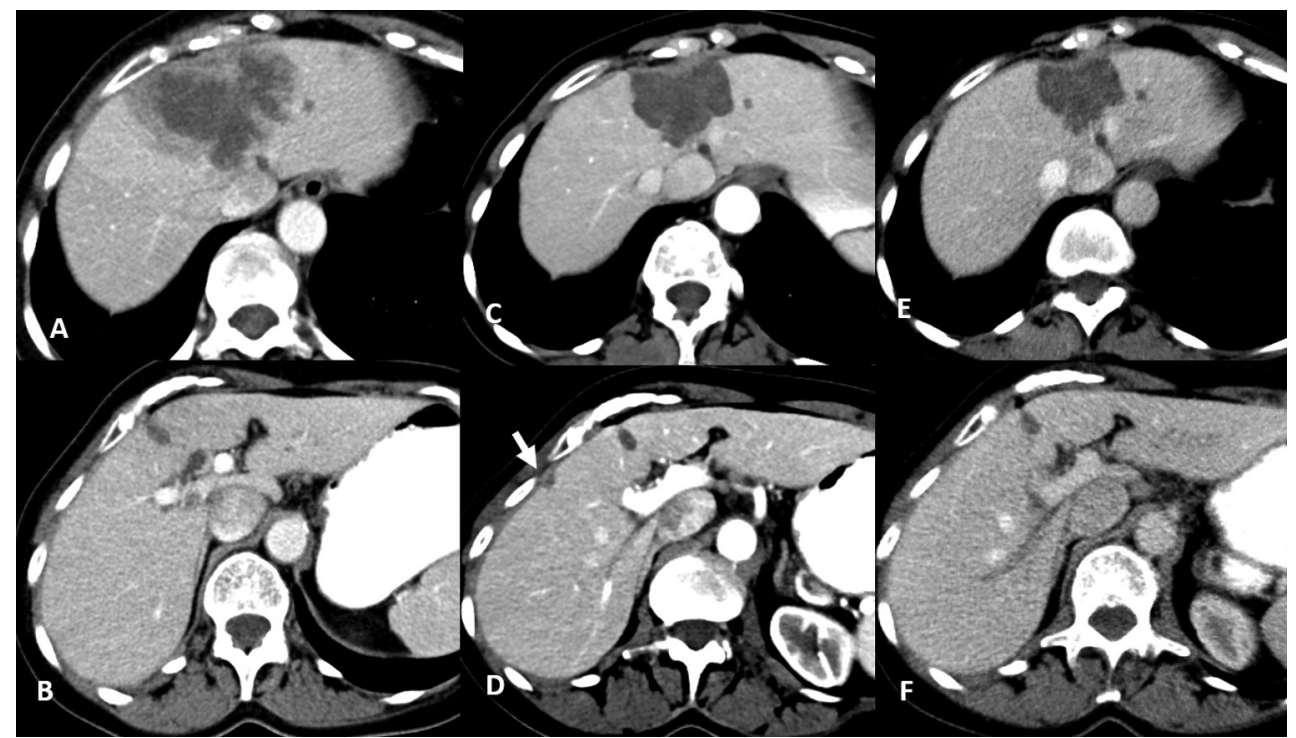

Fig. 2 The patient with metastatic melanoma. Baseline imaging (A, B) shows a peripherally enhancing lesion in segment VIII/IVa with central necrosis. No lesion is seen in seg V. Follow-up scan (C, D) 3 months after the patient received immunotherapy (ipilimumab and nivolumab) shows decrease in size of the seg VIII/IVa mass. However, a new subcapsular lesion is now seen in segment V (arrow in E). This was labeled iUPD (immune unconfirmed progressive disease). Imaging after 8 more weeks (E, F) reveals further decrease in the dominant mass that now appears predominantly cystic, with disappearance of the segment $V$ subcapsular lesion. This is consistent with iPR (immune partial response) according to iRECIST. 
irRC classifies four types of responses, depending on the time of the CT study vis-à-vis the patient response.

Type A: Decrease in size with no new lesions.

Type B: Stable disease that could remain unchanged or decrease in size on further follow-up.

Type C: Initial increase in the disease burden followed by response.

Type D: Decrease in disease burden despite appearance of new lesions.

If the initial posttherapy imaging demonstrates increased disease burden, a confirmatory scan at least 4 weeks later should be obtained to differentiate a type $C$ response from true progression.

Over the past decade, these criteria were often used in combination with the unidimensional RECIST or its modifications in various clinical trials. ${ }^{9}$ However, due to inhomogeneous application, the RECIST working group came out with immune RECIST (iRECIST) criteria for patients on immunotherapy. ${ }^{10}$ The chief features of iRECIST are as follows:

a. The definitions of measurable and nonmeasurable disease remain the same as RECIST 1.1 , as do the methods of measurement and imaging modalities for assessment.

b. If the patient has a type A or B response, it is assessed using RECIST 1.1.

c. A new category, namely unconfirmed progressive disease (iUPD-"i" standing for "immune"), has been introduced to account for type $\mathrm{C}$ or $\mathrm{D}$ response. If the initial posttherapy assessment reveals progressive disease, it is classified as iUPD. The next assessment must be done between 4 and 8 weeks after the scan that detects iUPD. This subsequent imaging may either reveal tumor shrinkage or stable disease, in which case the response is reclassified as iPR, iCR, or iSD, or demonstrate additional new lesions or increase in tumor size, in which case the response is reclassified as confirmed progressive disease (iCPD).

d. Thus, treatment should be continued even after detecting iUPD, until iCPD occurs.

\section{Cancer-Specific/Site-Specific Response Criteria} Assessment of Hepatocellular Carcinoma-EASL and modified RECIST

Unlike other tumors, locoregional therapies such as radiofrequency ablation and transarterial chemoembolization and targeted therapy in the form of sorafenib are the chief treatment modalities for HCC (apart from surgery). ${ }^{11,12}$ These treatments often lead to tumor necrosis without a significant decrease in tumor size (-Fig. 3); size may in fact occasionally increase due to intratumoral hemorrhage. The size-based WHO and RECIST criteria were thus not ideal in assessing tumor response in this setting. ${ }^{11,13,14}$ Hence, the EASL (European Association for the Study of the Liver) proposed ${ }^{14}$ the criteria in 2001 to assess tumor response based on the bidimensional WHO criteria, which incorporates measuring only the enhancing (viable) tumor component to assess response to therapy. Note that the portion of the tumor that demonstrated contrast enhancement on arterial phase $\mathrm{CT} /$ magnetic resonance imaging (MRI) was considered viable tumor. Subsequently, once RECIST criteria and unidimensional measurements got well established, the AASLD (American Association for the Study of Liver Disease) proposed modifications of RECIST criteria (modified RECIST [mRECIST]) based on the EASL criteria using unidimensional measurements. ${ }^{15}$ mRECIST also proposes to incorporate viable tumor volume and keeps cutoffs for progression and response similar to RECIST 1.1 (20\% and 30\%, respectively).

mRECIST clarifies the optimum imaging protocols for HCC, emphasizing on the need for obtaining quality liver imaging using CT or MRI, with at least dual-phase (arterial and portal venous phase) images acquired. The option to obtain delayed phase images is left to the institute based on its routine clinical practice; however, most institutes obtain delayed/equilibrium phase images as well.,16,17 mRECIST clarifies on various aspects of lesion selection and measurement. The salient features are discussed as follows:

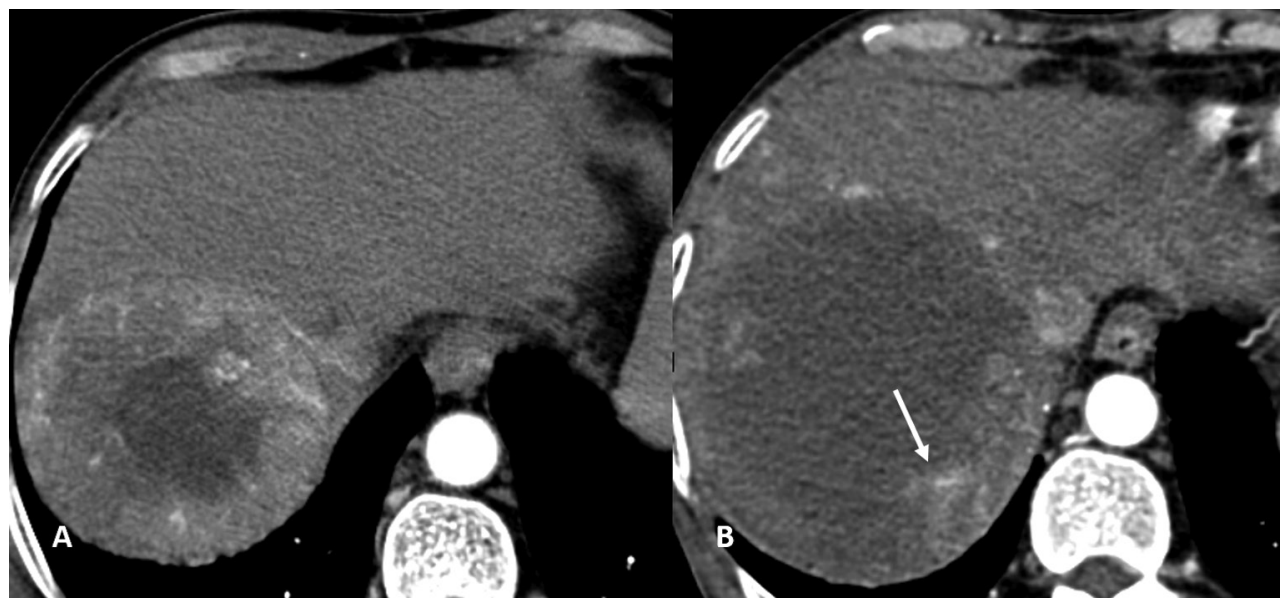

Fig. 3 The patient with hepatocellular carcinoma treated with transarterial chemoembolization (TACE). Axial arterial phase pre-TACE CT (A) demonstrates a large seg VII centrally necrotic mass with peripheral arterial enhancing viable tumor. This was treated with TACE. PostTACE arterial phase CT (B) shows near complete regression of the arterial enhancing viable tumor, with minimal residual enhancing soft tissue (arrow). Despite the mild increase in the lesion size, this corresponds to partial response (PR) as per mRECIST criteria. 
a. A target lesion at baseline should have at least a 1-cm arterial enhancing (viable) component in the longest dimension. It is important that the lesion be well defined and amenable to reproducible measurements. Ill-defined lesions such as infiltrative HCC should not be considered target lesions, nor should a malignant tumor thrombus.

b. As response is often in the form of variable necrosis, response assessment scans can measure the longest dimension of the tumor in a different plane from the baseline. No major areas of necrosis should be included while measuring the longest dimension.

c. For nontarget lesions as well, arterial enhancement and tumor necrosis are evaluated similar to target lesions. Thus, for example, complete resolution of arterial enhancing foci in non target lesions can be considered as complete response in the nontarget lesions.

d. Lymph nodes at porta hepatis should be considered malignant only if the short-axis diameter is at least $20 \mathrm{~mm}$. This is because reactive lymph nodes are commonly seen in patients with cirrhosis even in the absence of HCC, given that these patients have chronic hepatic inflammation.

e. mRECIST mandates cytopathologic confirmation of the malignant nature of any ascites of effusion that appears or worsens during treatment before being classified as progression, particularly when measurable disease shows response or is stable.

f. Appearance of a new HCC indicates disease progression. A new HCC is defined as a new arterial enhancing nodule $>1 \mathrm{~cm}$ in diameter, which also demonstrates washout. In absence of this typical vascular pattern, a new nodule > $1 \mathrm{~cm}$ in diameter can be diagnosed as HCC if it shows at least 1 -cm-interval growth on subsequent imaging. Other new lesions will be considered equivocal and appropriately followed up to assess for interval growth.

\section{Anderson Criteria for Bone}

Optimal imaging and response evaluation of bone metastases have always been a dilemma due to the complex structure and function of bone, with the possibilities of cortical or marrow involvement, and of osteoblastic or osteoclastic activation. Accordingly, bone metastases can be lytic, blastic, or mixed. Furthermore, bone metastases can be assessed both on structural imaging (CT, MRI, or X-rays) and on functional imaging (skeletal scintigraphy or ${ }^{18} \mathrm{~F}-\mathrm{FDG}$ PET that assesses osteoblastic activity or FDG-PET that assesses metabolic activity). Also, response to chemotherapy can manifest in the form of sclerotic changes in previously lytic metastases, whereas progression can manifest as development of new lytic foci in sclerotic or mixed metastases. ${ }^{17-19}$

The WHO criteria included response assessment of bone metastases ${ }^{20}$ based on radiographs. However, these were not included in the RECIST criteria, ${ }^{21}$ apart from assessment of an associated soft tissue component. Thus, patients with bone-only metastases could not be included in various clinical trials, as, for example, in metastatic breast cancer. Accordingly, Hamaoka et $\mathrm{a}^{22}$ from the MD Anderson Cancer Center proposed the MDA criteria for response assessment in bone metastases, which were based on the WHO criteria but incorporated skeletal scintigraphy, CT, and MRI into the assessment criteria. They found the MDA criteria to be better in distinguishing between responders and nonresponders as compared with WHO criteria, with the MDA criteria correlating with progression-free survival. ${ }^{9}$

Few important points to remember include the following ${ }^{12,22}$ :

1. Bone metastases may respond to therapy either by disappearing or by demonstrating a sclerotic response. Thus, complete or partial (usually involving rim) sclerotic change in a previously lytic lesion is usually a sign of response to therapy ( - Fig. 4). On the other hand, reappearance of a lucent focus in a previously sclerotic (responded) bony metastasis is suspicious for worsening disease.

2. On occasions, one or more previously inapparent lesions on CT may be seen as a "new" sclerotic focus on the restaging scan as they become easily visualized after sclerotic response. This should not be considered as a new lesion or disease progression, particularly if response is seen elsewhere.

3. Similarly, osteoblastic flare may be seen on scintigraphy, usually within the first 3 months of therapy. This occurs due to increased radiotracer uptake in the healing

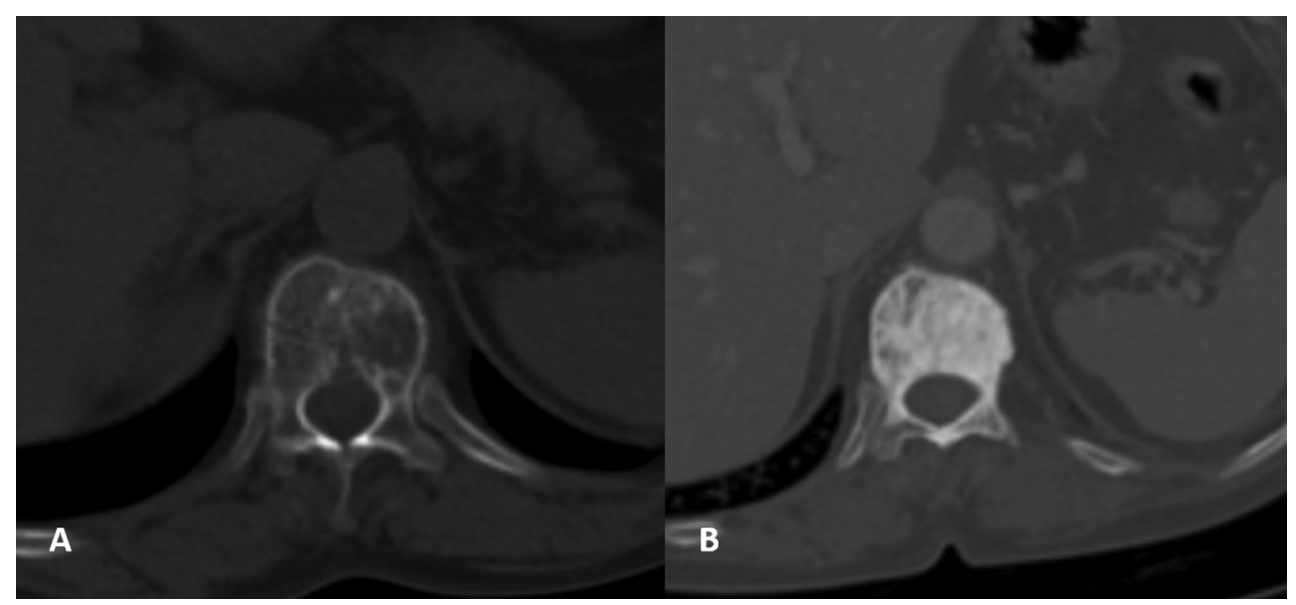

Fig. 4 Axial (A, B) CT images in bone window at 6 weeks interval. The lytic bony metastasis (A) has become sclerotic (B) on the follow-up scan, indicating response. Response assessment by MDA criteria indicates partial response in the vertebral metastasis. 
sclerotic lesions and should not be confused with disease progression.

4. Rapid osteolytic progression may be inadequately assessed as response on skeletal scintigraphy. In this setting, bony lesions may show decreased osteoblastic activity, resulting in apparent regression of "hot spots" on scintigraphy. X-rays or CT will help correctly identify progressive disease.

5. At follow-up, lesions seen at any of the imaging modalities should be compared with the images obtained at baseline, which most clearly define the bone lesions. The baseline images can be obtained on radiographs, CT, or MRI.

6. As a footnote, the MDA criteria are not often incorporated in clinical trials. However, it is important to remember these principles while assessing tumor response in routine patients, so as to avoid confusing bony response with progression.

\section{Functional Response Criteria}

The use of PET-CT in oncoimaging has grown tremendously in the past few years. However, PET-CT is not completely incorporated into the RECIST criteria. Accordingly, the PET Response Criteria In Solid Tumors (PERCIST) were proposed in 2009 to be used instead of RECIST in patients being assessed on PET-CT. Similarly, the Deauville criteria are commonly used in assessing lymphomas.

\section{PERCIST Criteria}

The rationale behind using FDG-PET for tumor response is that there is a strong positive relationship between tracer uptake and the number of viable cancer cells. ${ }^{23}$ As discussed previously, size-based criteria are not adequate to assess response to various targeted therapies and in certain organs. FDG-PET has a unique advantage of predicting response by assessing the change in tumor metabolism. Though it is congenial to assess the change in uptake qualitatively, this would raise the issue of reproducibility, thus limiting its use in clinical trials. One of the earliest attempts to address this issue was made by the European Organization for Research and Treatment of Cancer PET Study Group (EORTC criteria). ${ }^{24}$ After extensive review of the size-based criteria viz. WHO, RECIST and RECIST 1.1, and EORTC PET criteria, Wahl et $\mathrm{al}^{25}$ proposed PERCIST to harmonize a quantitative method of PET response that could be adopted in clinical practice and across various clinical trials. The PERCIST criteria are not meant to replace the RECIST criteria, but rather to complement them. They are expected to be valuable in prognosticating patients, otherwise labeled as "stable disease" by size-based criteria. ${ }^{26,27}$

Salient features of PERCIST include the following:

1. Scans should be performed in adequately prepared patients with well-calibrated and well-maintained scanner, and subsequent scans should be performed on the same scanner with similar doses of FDG and uptake time.

2. Peak standardized uptake value normalized to lean body mass (SULpeak) is used for assessment in PERCIST instead of widely used single pixel value, that is, SUVmax.

3. A 3-cm diameter spherical region of interest (ROI) generated over the normal liver is used as background activity and a 1-cm ROI over the thoracic aorta is used in case of a diseased liver. The background activity is used as quality control of the scan to assess the FDG avidity of tumor on the baseline scan.

4. Only one target lesion is selected, and the SULpeak is obtained by generating a $1.2-\mathrm{cm}$ diameter ROI around the hottest lesion. For a lesion to be eligible for PERCIST, the SULpeak of the baseline lesion must be greater than 1.5 times the liver SULpeak + 2 standard deviations. Note that unlike RECIST, PERCIST compares the hottest lesion of respective scans and not necessarily the same lesion.

5. PERCIST prefers to document change in tumor metabolism as a percentage change, and thus construct a waterfall plot. However, for convenience, tumor response can be grouped into categories as complete metabolic response (CMR) (-Fig. 5), partial metabolic response (PMR)

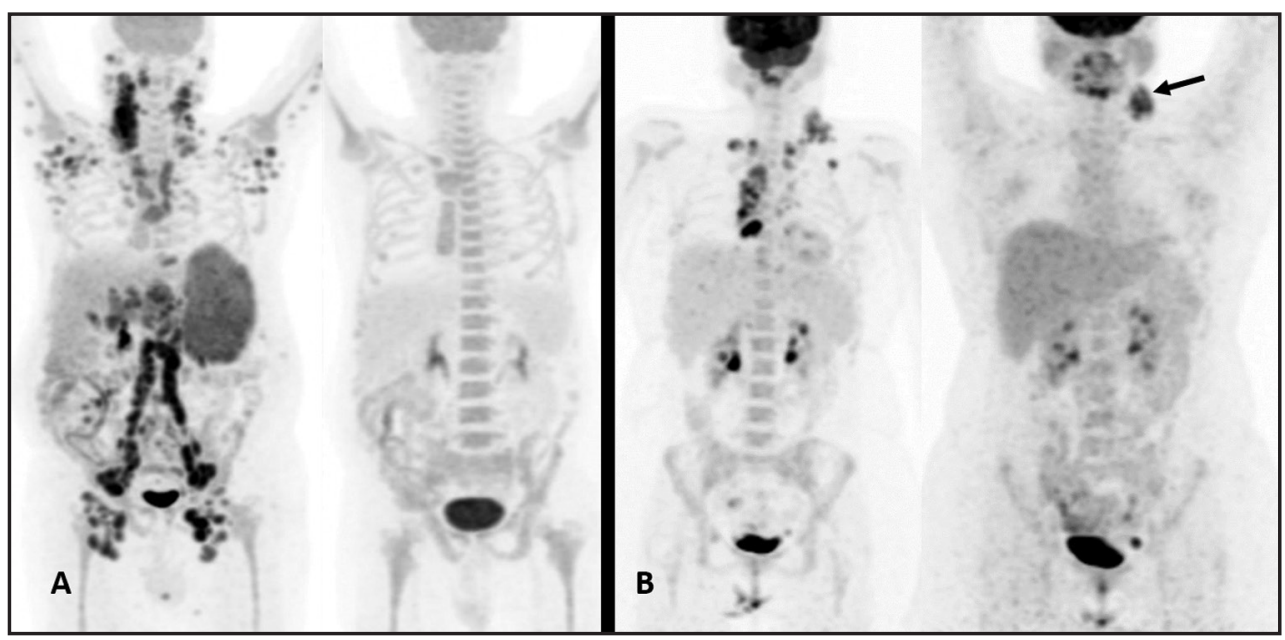

Fig. 5 Pre- and postchemotherapy maximum intensity projection (MIP) images (A) of a patient with diffuse large cell lymphoma show complete metabolic response (Deauville score 1) of the nodes and splenic lesions. Note that the diffuse uptake noted in marrow is resultant of stimulated marrow following chemotherapy and should not be misinterpreted as disease. Residual uptake more than liver (Deauville score 4 or 5 ) in the nodes (arrow) in the posttreatment scan is considered as residual disease (B). 


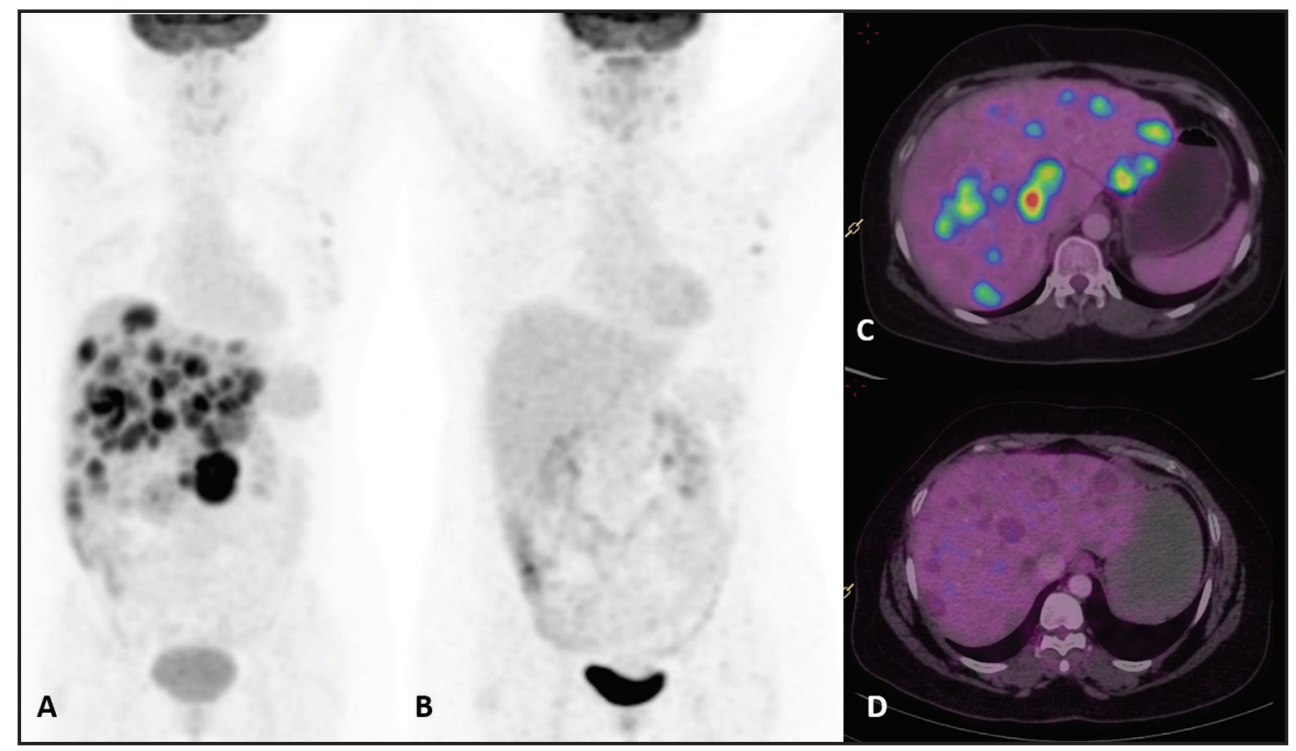

Fig. 6 Response assessment of a patient with gastrointestinal stromal tumor with liver metastases by PERCIST. Baseline MIP (A) and fused (C) images show hypermetabolic liver lesions and abdominal mass (arrow). PET-CT images (B, D) following 3 months of imatinib treatment show complete resolution of uptake in all the lesions suggesting CMR.

(-Fig. 6), stable metabolic disease (SMD), and progressive metabolic disease (PMD).

6. Authors of the PERCIST criteria acknowledge that it requires further validation and recommend to document additional parameters of up to five lesions to refine the criteria in future.

\section{Deauville Criteria}

Lymphoma is another disease that requires specific response evaluation criteria owing to complex disease process and newer targeted therapies. Often, the nodal mass does not regress completely even after completion of treatment without having any impact on outcome. ${ }^{28}$ Also, evaluating and predicting tumor response early during therapy could either spare toxic effects of "overtreatment" or warrant alternative treatment regimen in lymphoma. This has led to the emergence of interim (mid treatment) PET as a strong prognostic tool. One of the earliest criteria for PET response in lymphoma were the International Harmonized Project (IHP) criteria proposed in $2007.29 \mathrm{IHP}$ used mediastinal blood activity as threshold for deciding residual disease at the end of treatment. However, with the emergence of interim PET, mediastinal activity appeared to be a low threshold that caused false-positive scans. Subsequently new criteria was proposed in the "First International Workshop on interim-PET scan in lymphoma" held at Deauville, aptly called Deauville criteria for both interim and end-of-treatment assessments. ${ }^{30}$ It is a 5 -point (1-5) visual assessment scale (-Table 2). Tumor uptake moderately (score 4) or markedly (score 5) greater than liver uptake is considered as residual

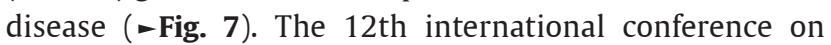
malignant lymphoma reinstated PET as indispensable for response assessment of FDG avid lymphomas and Deauville criteria as current standard assessment. ${ }^{31}$ That being said, we have not reached ideal criteria yet. There seems to be overlap between Deauville scores 3 and 4 as there is no quantitative parameter to differentiate them. Delta SUVmax
Table 2 Deauville 5-point scale

\begin{tabular}{|l|l|}
\hline Score & Grade of uptake \\
\hline 1 & No uptake \\
\hline 2 & Uptake $\leq$ mediastinum \\
\hline 3 & Uptake $>$ mediastinum and $\leq$ liver \\
\hline 4 & Uptake moderately increased above liver at any site \\
\hline 5 & $\begin{array}{l}\text { Markedly increased uptake above liver and/or new } \\
\text { sites of disease }\end{array}$ \\
\hline
\end{tabular}

and "Peking" criteria are some of the new criteria being explored. With more interim PET-based trials coming up, further refinement of the criteria can be expected in future.

\section{Other Criteria}

While we have covered the common alternative response criteria in the article, this review is by no means exhaustive. For example, other criteria such as RANO (Response Assessment in Neuro-Oncology) criteria for assessing brain tumors, ${ }^{32}$ MASS (Morphology, Attenuation, Size, and Structure) criteria for renal cell carcinoma, ${ }^{33}$ and tumor shrinkage criteria ( $10 \%$ criteria) for renal cell carcinoma ${ }^{34,35}$ have also been proposed. It is expected that with increasing understanding of tumor biology and with newer types of therapy emerging, more and more response patterns will be recognized and described.

\section{Take-Home Points for the Practicing Radiologist}

We have discussed the salient features of various alternative tumor response criteria in this article. It is again emphasized that though the criteria are meant for clinical trials, the principles expounded should be applied in routine clinical practice as well. For example,

1. First and foremost, it is important to be aware of the therapy that the patient is on. If there is any confusion 


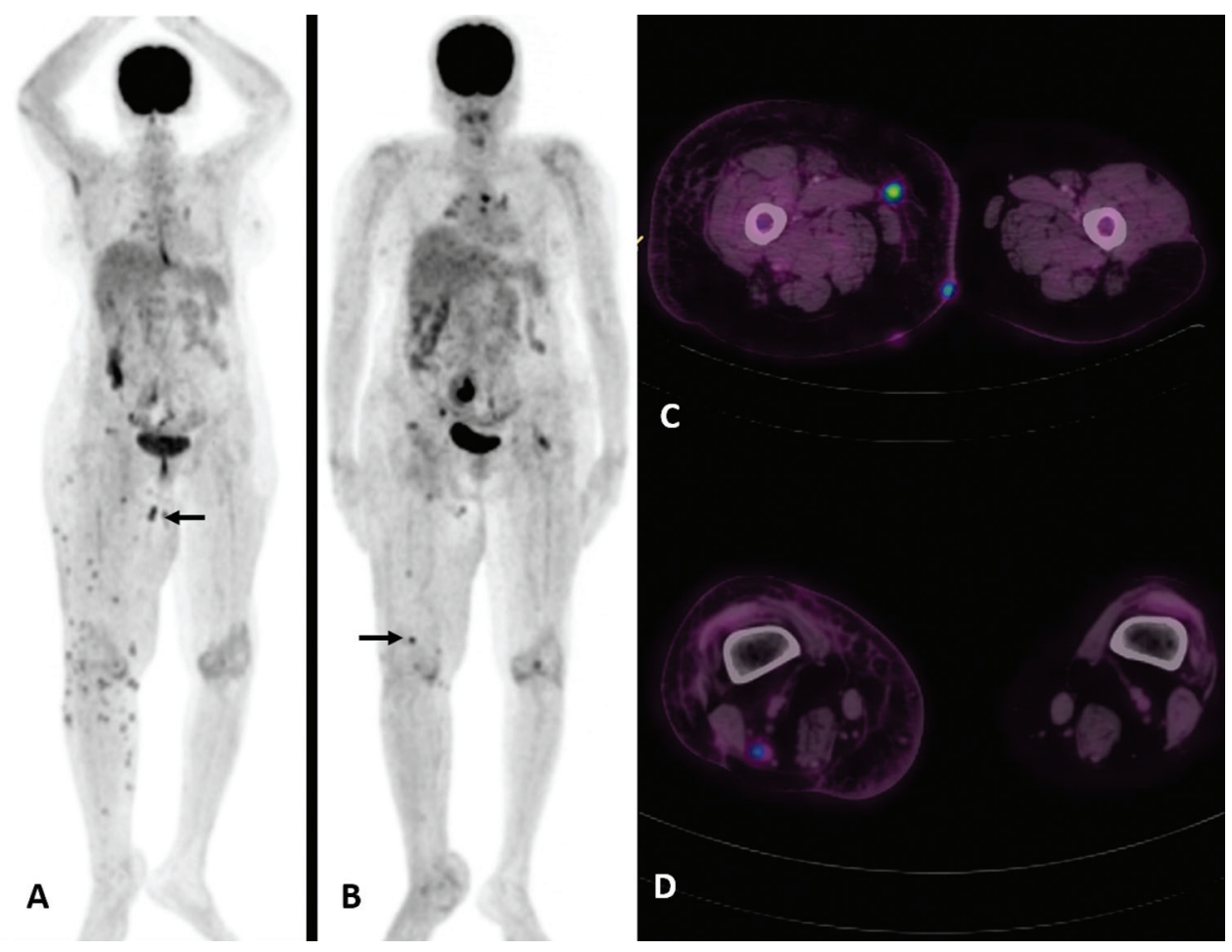

Fig. 7 Partial metabolic response of the patient with malignant melanoma according to PERCIST: SULpeak of the hottest lesion (arrow) in the baseline scan (A, C) was 14. Following 5 months of treatment, the SULpeak of the hottest lesion (block arrow) reduced to 8 , which was $>30 \%$ decrease, thus satisfying criteria for partial metabolic response. Note that hottest lesion in baseline and posttreatment scan may not be the same lesion.

regarding whether the patient is on targeted therapy or immunotherapy, always remember that the referring oncologist is only a phone call away!

2. Similarly, it is important to look at all relevant prior scans while reporting the current scan, be it a PET-CT or a bone scan.

3. Tumors may respond with altered morphology (as, e.g., decreased attenuation), particularly if not targeted therapy. This should be considered as a sign of response.

4. A patient on immunotherapy and demonstrating worsening disease or a new lesion may still be a responder (iUPD category), again highlighting the importance of knowing the patient's therapy before reporting the study.

5. HCC should be assessed based on the changes in the enhancing viable portion of the tumor.

6. Bone metastases often demonstrate a sclerotic response; this should not be confused as progression.

In conclusion, we have comprehensively discussed various alternative response criteria in this article. Understanding that each and every patient and cancer is unique, and that response assessment must be based on the patient's primary tumor, sites of metastases, and the treatment provided, is the key to delivering personalized radiology and precision medicine. Although complex, a basic understanding of the principles of various response criteria will enable the practicing radiologist to function as an important component of the multidisciplinary oncology team and add value to patient management.

\section{Conflict of Interest}

None declared.

\section{Acknowledgment}

The authors thank Dr. Nilendu Purandare, Tata Memorial Hospital, New Delhi, for providing his guidance and expertise in the writing and review of the manuscript.

\section{References}

1 Tirumani SH, Baheti AD, Tirumani H, O'Neill A, Jagannathan JP. Update on gastrointestinal stromal tumors for radiologists. Korean J Radiol 2017;18(1):84-93

2 Tirumani SH, Jagannathan JP, Krajewski KM, Shinagare AB, Jacene H, Ramaiya NH. Imatinib and beyond in gastrointestinal stromal tumors: a radiologist's perspective. Am J Roentgenol 2013;201(4):801-810

3 Choi H. Response evaluation of gastrointestinal stromal tumors. Oncologist 2008;13(Suppl 2):4-7

4 Gonzalez-Guindalini FD, Botelho MPF, Harmath CB, et al. Assessment of liver tumor response to therapy: role of quantitative imaging. Radiographics 2013;33(6):1781-1800

5 Nishino M, Tirumani SH, Ramaiya NHHF, Hodi FS. Cancer immunotherapy and immune-related response assessment: the role of radiologists in the new arena of cancer treatment. Eur J Radiol 2015;84(7):1259-1268

6 Choi Y, Kim DH, Jin SY, Lee AY, Lee SH. Topical immunotherapy with diphenylcyclopropenone is effective and preferred in the treatment of periungual warts. Ann Dermatol 2013;25(4):434-439

7 O'Regan KN, Jagannathan JP, Ramaiya N, Hodi FS. Radiologic aspects of immune-related tumor response criteria and patterns of immune-related adverse events in patients undergoing ipilimumab therapy. Am J Roentgenol 2011;197(2): W241-6

8 WolchokJD, Hoos A, O'Day S, et al. Guidelines for the evaluation of immune therapy activity in solid tumors: immune-related response criteria. Clin Cancer Res 2009;15(23):7412-7420 
9 Nishino M, Jagannathan JP, Krajewski KM, et al. Personalized tumor response assessment in the era of molecular medicine: cancer-specific and therapy-specific response criteria to complement pitfalls of RECIST. Am J Roentgenol 2012;198(4):737-745

10 Seymour L, Bogaerts J, Perrone A, et al; RECIST working group. iRECIST: guidelines for response criteria for use in trials testing immunotherapeutics. Lancet Oncol 2017;18(3):e143-e152

11 Llovet JM, Ducreux M, Lencioni R, et al; European Association For The Study Of The Liver; European Organisation For Research And Treatment Of Cancer. EASL-EORTC clinical practice guidelines: management of hepatocellular carcinoma. J Hepatol 2012;56(4):908-943

12 Subbiah V, Chuang HH, Gambhire D, Kairemo K. Defining clinical response criteria and early response criteria for precision oncology: current state-of-the-art and future perspectives. Diagnostics (Basel) 2017;7(1):10

13 Arslanoglu A, Seyal AR, Sodagari F, et al Current guidelines for the diagnosis and management of hepatocellular carcinoma: a comparative review. Am J Roentgenol 2016;207(5):W88-W98

14 Bruix J, Sherman M, Llovet JM, et al; EASL Panel of Experts on HCC; European Association for the Study of the Liver. Clinical management of hepatocellular carcinoma. Conclusions of the Barcelona-2000 EASL conference. J Hepatol 2001;35(3):421-430

15 Llovet JM, Di Bisceglie AM, Bruix J, et al; Panel of Experts in HCC-Design Clinical Trials. Design and endpoints of clinical trials in hepatocellular carcinoma. J Natl Cancer Inst 2008;100(10):698-711

16 Lencioni R. New data supporting modified RECIST (mRECIST) for hepatocellular carcinoma. Clin Cancer Res 2013;19(6):1312-1314

17 Woolf DK, Padhani AR, Makris A. Assessing response to treatment of bone metastases from breast cancer: what should be the standard of care? Ann Oncol 2015;26(6):1048-1057

18 Costelloe CM, Chuang HH, Madewell JE, Ueno NT. Cancer response criteria and bone metastases: RECIST 1.1, MDA and PERCIST. J Cancer 2010;1(1):80-92

19 Schwartz LH, Seymour L, Litière S, et al. RECIST 1.1-standardisation and disease-specific adaptations: perspectives from the RECIST Working Group. Eur J Cancer 2016;62(March): 138-145

20 WHO Handbook for Reporting Results of Cancer Treatment. Vol. 48, World Health Organization Offset Publication; 1979

21 Eisenhauer EA, Therasse P, Bogaerts J, et al. New response evaluation criteria in solid tumours: revised RECIST guideline (version 1.1) Eur J Cancer 2009;45(2):228-247

22 Hamaoka T, Madewell JE, Podoloff DA, Hortobagyi GN, Ueno NT. Bone imaging in metastatic breast cancer. J Clin Oncol 2004;22(14):2942-2953

23 Bos R, van Der Hoeven JJ, van Der Wall E, et al. Biologic correlates of (18)fluorodeoxyglucose uptake in human breast cancer measured by positron emission tomography. J Clin Oncol 2002;20(2):379-387

24 Young H, Baum R, Cremerius U, et al; European Organization for Research and Treatment of Cancer (EORTC) PET Study Group. Measurement of clinical and subclinical tumour response using [18F]-fluorodeoxyglucose and positron emission tomography: review and 1999 EORTC recommendations. Eur J Cancer 1999;35(13):1773-1782

25 Wahl RL, Jacene H, Kasamon Y, Lodge MA. From RECIST to PERCIST: evolving considerations for PET response criteria in solid tumors. J Nucl Med 2009;50(1, Suppl 1):122S-150S

26 Min SJ, Jang HJ, Kim JH. Comparison of the RECIST and PERCIST criteria in solid tumors: a pooled analysis and review. Oncotarget 2016;7(19):27848-27854

27 Shang J, Ling X, Zhang L, et al. Comparison of RECIST, EORTC criteria and PERCIST for evaluation of early response to chemotherapy in patients with non-small-cell lung cancer. Eur J Nucl Med Mol Imaging 2016;43(11):1945-1953

28 Jochelson M, Mauch P, Balikian J, Rosenthal D, Canellos G. The significance of the residual mediastinal mass in treated Hodgkin's disease. J Clin Oncol 1985;3(5):637-640

29 Juweid ME, Stroobants S, Hoekstra OS, et al; Imaging Subcommittee of International Harmonization Project in Lymphoma. Use of positron emission tomography for response assessment of lymphoma: consensus of the Imaging Subcommittee of International Harmonization Project in Lymphoma. J Clin Oncol 2007;25(5):571-578

30 Meignan M, Gallamini A, Meignan M, Gallamini A, Haioun C. Report on the First International Workshop on interim-PET scan in lymphoma. Leuk Lymphoma 2009;50(8):1257-1260

31 Cheson BD, Fisher RI, Barrington SF, et al; Alliance, Australasian Leukaemia and Lymphoma Group; Eastern Cooperative Oncology Group; European Mantle Cell Lymphoma Consortium; Italian Lymphoma Foundation; European Organisation for Research; Treatment of Cancer/ Dutch Hemato-Oncology Group; Grupo Español de Médula Ósea; German High-Grade Lymphoma Study Group; German Hodgkin's Study Group; Japanese Lymphorra Study Group; Lymphoma Study Association; NCIC Clinical Trials Group; Nordic Lymphoma Study Group; Southwest Oncology Group; United Kingdom National Cancer Research Institute. Recommendations for initial evaluation, staging, and response assessment of Hodgkin and non-Hodgkin lymphoma: the Lugano classification. J Clin Oncol 2014;32(27):3059-3068

32 Chang SM, Wen PY, Vogelbaum MA, Macdonald DR, van den Bent MJ. Response Assessment in Neuro-Oncology (RANO): more than imaging criteria for malignant glioma: . Neurooncol Pract 2015;2(4):205-209

33 Smith AD, Shah SN, Rini BI, Lieber ML, Remer EM. Morphology, Attenuation, Size, and Structure (MASS) criteria: assessing response and predicting clinical outcome in metastatic renal cell carcinoma on antiangiogenic targeted therapy. Am J Roentgenol 2010;194(6):1470-1478

34 Krajewski KM, Guo M, Van den Abbeele AD, et al. Comparison of four early posttherapy imaging changes (EPTIC; RECIST 1.0, tumor shrinkage, computed tomography tumor density, Choi criteria) in assessing outcome to vascular endothelial growth factor-targeted therapy in patients with advanced renal cell carcinoma. Eur Urol 2011;59(5):856-862

35 Arya S, Das D, Engineer R, Saklani A. Imaging in rectal cancer with emphasis on local staging with MRI. Indian J Radiol Imaging 2015;25(2):148-161 